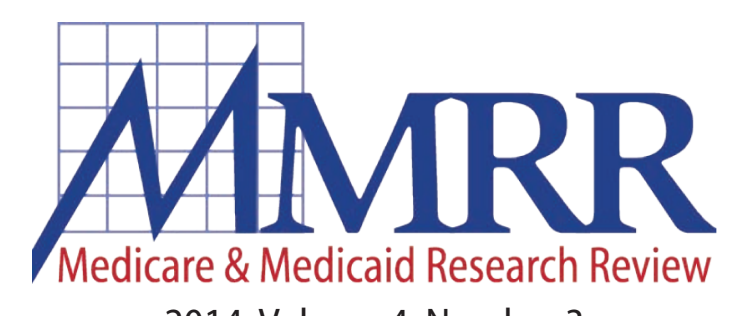

2014: Volume 4, Number 3

A publication of the Centers for Medicare \& Medicaid Services, Office of Information Products \& Data Analytics

\title{
HAC-POA Policy Effects on Hospitals, Other Payers, and Patients
}

\author{
Asta Sorensen, Nikki Jarrett, Elizabeth Tant, \\ Shulamit Bernard, and Nancy McCall \\ RTI International
}

Background: Prior to the implementation of the Hospital-Acquired Condition-Present on Admission (HAC-POA) payment policy, concerns regarding its potential impact were raised by a number of organizations and individuals. The purpose of this study was to explore direct and indirect effects of the HAC-POA payment policy on hospitals, patients, and other payers during the policy's first 3 years of implementation.

Methods: The study included semi-structured telephone interviews with representatives of national organizations, hospitals, patient advocacy organizations, and other payers. Interview notes were coded using QSR NVivo qualitative analysis software using inductive and deductive qualitative analysis techniques.

We conducted interviews with 106 individuals representing 56 organizations. Hospital staff included physicians, nurses, patient safety officers, coders, and finance, senior management, and information management staff. Individuals from other organizations represented leadership positions.
Results: Key changes to hospitals included: cultural shifts involving attention, commitment, and support from hospital leadership for patient safety; hiring new staff to assure the accuracy of clinical documentation and POA oversight structures; increased time burden for physicians, nurses, and coders; need to upgrade or purchase new software; and need to collaborate with hospital departments or staff that did not interface directly in the past. The policy was adopted by a majority of other payers, although the list of conditions and payment penalties varies. The HAC-POA policy is invisible to patients; therefore, the presence or lack of unintended consequences to patients cannot be fully assessed at this time. Understanding of policy effects to all stakeholders is important for maximizing its successful implementation and desired impact.

Keywords: qualitative research, Medicare, hospitals, health policy, politics, law, regulation, evaluation design and research

ISSN: 2159-0354

doi: http://dx.doi.org/10.5600/mmrr.004.03.a07 
Medicare \& Medicaid Research Review 2014: Volume 4, Number 3

\section{Mission Statement}

Medicare \& Medicaid Research Review is a peerreviewed, online journal reporting data and research that informs current and future directions of the Medicare, Medicaid, and Children's Health Insurance programs. The journal seeks to examine and evaluate health care coverage, quality and access to care for beneficiaries, and payment for health services.

\section{http://www.cms.gov/MMRR/}

\section{Centers for Medicare \& Medicaid Services Marilyn Tavenner Administrator}

Editor-in-Chief

David M. Bott, Ph.D.

The complete list of Editorial Staff and Editorial Board members

may be found on the MMRR Web site (click link): $\underline{\text { MMRR Editorial Staff Page }}$

Contact: $\underline{\text { mmrr-editors@cms.hhs.gov }}$

Published by the Centers for Medicare \& Medicaid Services.

All material in the Medicare \& Medicaid Research Review is in the public domain and may be duplicated without permission. Citation to source is requested.

\section{Introduction}

Introduction of the Hospital-Acquired ConditionPresent on Admission (HAC-POA) payment policy by the Centers for Medicare \& Medicaid Services (CMS) raised a number of concerns about its potential impact on hospitals, patients, and other payers. These concerns were largely related to the potential for unnecessary diagnostic testing of patients (Wald \& Kramer 2007; Saint, Meddings, Calfee, Kowalski, \& Krein, 2009); diversion of personnel and other resources away from general patient care and prevention leading to additional time, personnel, and resource consumption (Brown, Doloresco, \& Mylotte, 2009; McHugh, Martin, Orwat, \& Dyke, 2011; McHugh, Van Dyke, Osei-Anto, \& Haque, 2011); and reduction or changes in health care worker staffing due to decreased reimbursement (Parish 2008; Brown et al.,2009). At least one source suggested that public disclosure of HAC rates would help patients make better-informed health care choices (McKibben et al., 2005). Patient advocates and others raised concerns about reduced access to care for high-risk patients, denial of care or substandard care due to decreased reimbursement, and increased out-ofpocket costs for patients. Many stakeholders also anticipated a positive effect: that the financial impact of the HAC-POA payment policy could cause hospital executives to redefine priorities and embrace efforts toward improved patient care (Rosenstein, O'Daniel, White, \& Taylor, 2009; Stone et al., 2010), resulting in improved care and hospital acquired infection (HAI) rate reductions.

Following policy implementation, a number of studies have assessed the impact of the HACPOA policy with respect to HAC outcomes, impact on hospital staffing, practices, and costs. Several studies have undertaken assessment of the 
impact of the HAC-POA policy on the incidence of specific HACs based on data from medical claims, the National Healthcare Safety Network (NHSN), or discharge records (Lee, Kleinman, et al., 2012; Meddings, Reichert et al., 2012; Peasah, McKay, Harman, Al-Amin, \& Cook, 2013). Other studies assessed the impact qualitatively by focusing on feedback from hospital staff: infection preventionists (Hoff et al., 2011; Hartmann et al., 2012; Lee, Hartmann, et al., 2012), safetynet hospital chief quality and financial officers (McHugh, Van Dyke et al., 2011), chief nursing officers (Wald, Richard, Dickson, \& Capezuti, 2012), or hospital infection and quality control personnel (Stone, et al., 2011). However, no studies to date provide a comprehensive understanding of policy effects to a broader spectrum of key stakeholders. A better understanding of unintended consequences-positive or negative-and spillover effects is essential for successful implementation of the policy and potential future refinements.

The purpose of this study was to identify direct and indirect HAC-POA policy effects (including unintended consequences and spillover) on hospitals and payers during the first three years following implementation, as reported by a wide range of healthcare and insurer personnel, and also to explore the impact of the policy on patient experiences.

\section{Methods}

Our methodological approach included semi-structured telephone interviews with representatives of national organizations and state hospital associations, hospitals, professional coding organizations, patient advocacy organizations, and other payers (state Medicaid programs and private insurers). Hospital staff included physicians, nurses, patient safety officers, coders, finance, senior management, and information management staff. Individuals from other organizations represented leadership positions; i.e., Executive Directors, Medical Directors, Vice President for Quality and Patient Safety.

The study was conducted during September 2009-September 2012 in three phases. During the first phase (October 2009-September 2010) and the first year after the policy went into effect, we sought to obtain early experiences with policy implementation from hospitals, national hospital, data, or coding organizations, and other payers. During the second phase (October 2010-September 2011), we targeted hospitals and patient advocacy groups. During the third phase (October 2011-September 2012), we sought to obtain additional feedback about implementation experience from state hospital associations and coding professionals, and to learn about spillover effects from the private payers.

Candidate national and state hospital, patient advocacy, and other payer organizations were identified using purposive sampling techniques by targeting organizations that would be most likely affected by HAC-POA policy, either directly or through their constituents. Interview participants were identified by contacting individuals in senior or most relevant positions and asking for their recommendations for individuals within their organizations who were most knowledgeable about HAC-POA policy implementation or its effects. Each candidate organization was contacted by e-mail up to three times. To allow for data saturation, we sought to identify up to nine organizations in each respondent category. Interviews were conducted individually or in groups, based on interviewee designation and preference.

Because payment reduction can be a strong driver of hospital strategies pertaining to policy compliance, hospital selection criteria during the 
second phase of the study were based on payment impact. We used the first 9 months of Fiscal Year 2009 MedPAR files (2010 data were not available at that time) and selected 17 hospitals that had the highest aggregate payment reductions and 17 hospitals that had minimal or no reductions, with the goal to over-recruit and achieve a final sample of up to 9 hospitals in each of the two categories. Hospitals that had minimal or no reductions were selected randomly, selecting each $5^{\text {th }}$ hospital from the list sorted by amount of payment penalty. Whenever possible, we sought to have a balanced representation of hospitals in this category based on size and location and made a replacement with a hospital that had the same impact in payment penalties and brought more diversity in terms of size and location characteristics. We limited the affiliation of each selected hospital to one unique healthcare system so that no more than one hospital was affiliated with the same health care system.

Discussion guides were tailored to each type of organization and interviewee role. Interviews with hospital staff and national or state hospital organizations centered on organizational changes that had to be made to assure the compliance with the HAC-POA policy; strategies that hospitals took in order to meet HAC-POA policy requirements; policy impact on patient care; policy impact on the cost of care; changes in financial performance and efficiency; changes in HAC incidence; policy effects on admission, discharge, diagnostic, and treatment patterns; changes to hospital infrastructure, operations, policies and procedures, staffing, and overall performance; POA coding practices, and policy impact to non-Medicare patients.

Interviews with patient advocacy groups sought to learn about patient experiences with the HAC-POA policy; obtain feedback received by patient advocacy organizations about HACPOA policy from patients; learn ways in which the policy might have affected patient experiences; learn ways in which the policy changed patient care, admission, discharge, diagnosis, treatment patterns, and provider performance; understand the policy impact on non-Medicare patients; learn ways in which the policy improved patient safety; and gain recommendations for policy refinements from patients' perspective.

Interviews with other payers focused on spillover effects, such as reasons for implementing policies similar to CMS HAC-POA; similarities and differences between the policies; ways that other payers address POA; financial impact of the policy; HAC incidence at the contracted hospitals; policy effects to the contracted hospitals; strategies to assess accuracy of coding; challenges experienced with the policy; and anticipated policy changes, modifications, and future directions.

Telephone interviews for all three groups were conducted by two researchers trained in qualitative interviewing and research techniques. Each interview lasted about an hour. Prior to each interview, we obtained informed consent to participate in the interview and to allow for audio-recording. A note-taker accompanied each interview and took notes in real time.

The study received exempt status from the Institutional Review Board of RTI International.

\section{Analysis}

Interview notes were finalized immediately after the interview, using audio recording to verify the accuracy of captured data. The notes were imported to QSR NVivo qualitative analysis software for coding.

The list of codes were developed iteratively, following each phase of the interviews. Early analyses were more exploratory in nature (Patton, 1990). Codes were developed based on interview questions, review of literature, initial review of interviews, and additional emerging 
categories. Emergent themes served as working hypotheses that were tested against the data from all interviews. Subsequent analyses for the second and third phases were more confirmatory, deepening the insights and confirming or disconfirming themes that emerged during the prior phases (Patton, 1990; Miles \& Huberman, 1994). A team of three researchers trained in qualitative analysis techniques participated in the coding process, the review and discussions of code outputs, and the summary of results.

\section{Results}

In this section, we first report the number of individuals, and their affiliate organizations, who participated in key informant interviews, followed by a report of participant responses addressing direct and indirect effects of the policy on hospitals, other payers, and patients.

\section{Interviewees}

We conducted interviews with 106 individuals representing 56 organizations. Exhibit 1 summarizes the type and number of participating organizations and interviewees. Hospitals represented a variety of geographic regions, sizes, and teaching statuses. The range of hospital sizes and HAC incidence is listed in Exhibit 2.

Seven hospitals or healthcare systems participated in interviews during Phase 1. Hospitals contacted during the second phase of the study were reluctant to participate in the interviews. We contacted 34 hospitals and received no response from 22 hospitals (response rate of 35\%) after at least three repeated attempts to contact Chief Executive Officers (CEOs) and/or quality and patient safety officers. Representatives from two organizations said they had no time to provide feedback given that participation
Exhibit 1. Number of Participating Organizations and Interviewees

\begin{tabular}{lcc}
\hline $\begin{array}{l}\text { Types of } \\
\text { organizations }\end{array}$ & $\begin{array}{c}\text { Number of } \\
\text { organizations }\end{array}$ & $\begin{array}{c}\text { Number of } \\
\text { interviewees }\end{array}$ \\
\hline Hospitals & 17 & 41 \\
National & 5 & 9 \\
$\begin{array}{l}\text { Organizations } \\
\text { Other Payers }\end{array}$ & 14 & 23 \\
Patient & 4 & 4 \\
Advocacy & & \\
$\begin{array}{l}\text { Organizations } \\
\text { Professional }\end{array}$ & 7 & 10 \\
$\begin{array}{l}\text { Coding } \\
\text { Organizations }\end{array}$ & & \\
Hospital & 9 & 19 \\
$\begin{array}{l}\text { Associations } \\
\text { Total }\end{array}$ & 56 & 106 \\
\hline
\end{tabular}

Exhibit 2. Description of Participating Hospitals

\begin{tabular}{lcc}
\hline Name & Size (beds) & HAC-Incidence \\
\hline H1 & 98 & Low \\
H2 & 373 & Low \\
H3 & 159 & Low \\
H4 & 91 & Low \\
H5 & 333 & Low \\
H6 ${ }^{1}$ & NA & NA \\
H7 ${ }^{2}$ & NA & NA \\
H8 & 289 & High \\
H9 & 501 & High \\
H10 & 540 & High \\
H11 & 636 & High \\
H12 & 276 & Low \\
H13 & 289 & Low \\
H14 & 301 & Low \\
H15 & 568 & Low \\
H16 & 128 & Low \\
H17 & 99 & Low \\
\hline NOTES: ${ }^{1}$ H6-Represents health care system responsible for \\
130 hospitals. \\
${ }^{2}$ H7-Represents health care system responsible for \\
27 hospitals. \\
SOURCE: Authors' data combined with corresponding 2009 \\
MedPAR files ${ }^{2}$ ata. & &
\end{tabular}


was not mandatory; two hospitals declined to participate in the interviews because of concerns of confidentiality. Of 31 contacted hospital coders, 17 declined participation, citing a lack of knowledge regarding the impact of policy within their organizations.

Two of six patient advocacy organizations declined to participate because of a lack of patient feedback about HAC-POA policy from their constituents. Likewise, two state hospital associations declined to participate, citing lack of knowledge regarding the policy implementation or impact among the hospitals in their states. Thirtyfive other payer organizations were contacted, 14 of which participated in the interviews.

\section{Effects on Hospitals}

We observed no differences in HAC-POA policy implementation experiences or impact between hospitals who had the highest aggregate payment reductions and those who had minimal or no reductions. Key HAC-POA policy effects on hospitals centered on organizational culture and teamwork, staffing and infrastructure, and care and treatment patterns.

\section{Organizational Culture and Teamwork}

Most patient advocates, hospital staff, and representatives of national hospital associations concurred that key organizational culture changes attributable to the HAC-POA policy were attention, commitment, and support from hospital leadership for patient safety. Before the policy, leadership commitment to patient safety was limited to a handful of leading performers in the country. Additionally, safety initiatives within hospitals were limited to isolated units and individuals. After introduction of HAC-POA policy, patient safety became a priority to every hospital, every unit, and every provider.

Some hospital staff noted that compliance with the policy facilitated teamwork and brought traditionally dispersed departments closer together (e.g., finance, nursing, coding, and leadership). Others, however, suggested that challenges pertaining to documentation and coding issues might have placed further strains in relationships between nurses and physicians in the process of assessment, staging, documentation, and coding accuracy of pressure ulcers as well as accuracy of POA assessments for the rest of the HACs.

\section{Staffing and Infrastructure}

Most hospital staff stated that HAC-POA policy implementation resulted in time, personnel, and resource consumption, such as the need to hire clinical documentations specialists or changing the work flows to establish oversight structures, such as second reviews or committees. Changes in hiring practices included increased hiring of clinical documentation specialists. In some hospitals, such a role existed before the HACPOA policy; others created new positions after the policy took effect. Since the policy took place, a typical mid-sized hospital reported having four to seven documentation specialists with clinical backgrounds and knowledge of Medicare severity diagnosis-related groups (MS-DRGs). Larger hospitals reported having as many as 12 documentation specialists. Much of their role is to ensure the accuracy of documentation through a review of new admissions and to ensure that physicians have accurately documented the situation and treatment plan, which, in turn, allows for more accurate coding. Some hospitals have expanded the role of nurse case managers to include documentation and coding oversight for POA conditions.

... They may go right back to the physician and ask questions, but, typically, by the time they get around to coding a chart, the patient has been discharged, so it's pretty hard 
to change the documentation at that point to indicate POA. We try to do this in real time because if not, it's hard to go back and amend the chart to say it was POA and it just looks bad in the chart.

In views of most hospital staff, the policy increased physician and nursing staff time for completing discharge summaries and reconciling POA queries. Likewise, hospitals' efforts to comply with HAC-POA decreased productivity on clinical staff and coders:

We've had to have more queries and do more physician education and that's sometimes a source of friction between coders and physicians. Physicians are busy people and they're done with this patient and onto the next, then we come back two weeks later and ask them a question about something they have already moved beyond. The query process can be difficult, so we've had to do more queries, which means more follow up with physicians as well, so that adds a lot of time and stress.

In addition to assigning codes for various diagnoses and procedures, they now have to look at whether it was POA and enter that information. If it's not clear whether it was POA, they have to go back and query the physician. It definitely has added to their workload and had an impact on [coders'] productivity.

Most hospital staff reported that their hospitals adopted guidelines, developed toolkits, and engaged in extensive training for POA identification. Most such changes took place with the initial introduction of policy during the first year and did not require on-going time investment. Changes in billing practices entailed the use of new financial and billing software and the enactment of new policies for non-billing of preventable HACs. Changes in codes and coding requirements created the need for software modifications, new software, and training.

\section{Care and Treatment}

Most hospital staff shared having greater adherence to evidence-based guidelines (EBGs); new protocols for prophylactic care treatment; and increased focus on prevention (e.g., providing sitters to prevent patient falls; testing for infections at admission); implementation of safety procedures for HAC prevention; changes in testing protocols for urinary tract infections (UTIs); and development of updated protocols and physician guides. Many hospitals incorporated verification of POA and HAC status into their quality reviews and internal audits. Some shared that they hired external consultants to conduct coding and financial audits.

\section{HAC Incidence and Tracking}

Only a few hospital interviewees reported observing specific changes in HAC incidence patterns at their organizations. Some suggested that such trends were not meaningful to the hospitals because many hospital staff believe that HAC-POA data lack face validity, do not provide useful measures, and do not allow meaningful and valid comparisons across or within organizational performance over time. Most hospital interviewees shared that they generally preferred Centers for Disease Control and Prevention (CDC) reporting systems, definitions, and data for infections, which they found more helpful for internal improvement purposes. Inconsistent definitions used by CDC and CMS 
were viewed as a source of misunderstandings and decreased accuracy of coding.

Staff of most hospitals reported notable improvements in pressureulcer, catheter-associated UTI (CAUTI), and Vascular Catheter-Associated Infection rates because of improvements in pressure ulcer identification and staging practices, as well as CAUTI prevention and tracking as a result of HAC-POA. Staff from one or two hospitals noted that there had been an increase in urinalysis culture for patients admitted to their facilities with indwelling catheters.

Hospital interviewees identified few concerns pertaining to HACs that are rare (or "never") events (e.g., blood incompatibility). Key concerns for certain HACs were lack of effective EBGs and dependence on patient compliance or comorbidities, which hampered hospitals' ability to avoid some of the HACs.

Coding challenges unique to specific HACs include difficulties in identification and documentation of specific HACs, including Pressure Ulcers Stages III \& IV, CAUTIs, DVT, and Pulmonary Embolism Following Certain Orthopedic Procedures. Several nursing staff shared that it was difficult to distinguish an infection from colonization for UTIs. Coding challenges stemmed from reliance on physician notes for some conditions traditionally identified by nurses, particularly for pressure ulcers, difficulties in determining POA, and the need for coder/physician interaction or other method of verification to ensure accuracy. Hospital interviewees shared that, although they were documenting HACs and POA prior to the implementation of the HAC-POA policy, they are documenting more thoroughly since the policy implementation. They also reported the challenge of finding a balance between clinical documentation and administrative documentation and were concerned that these efforts derailed the attention of clinical staff from direct patient care.

\section{Effects on Other Payers}

The HAC-POA policy had spillover effects on other insurers. Private insurers have enacted similar payment policies, although the list of conditions varies. Private payers shared that their HAC-POA policies were driven by the national agenda set by CMS. Interviewees representing private payers reported that HAC-POA policies were receiving a lot of interest from employer organizations:

Employer organizations want us to do even more every single day. ... Employers do want a report of POA indicators on their employees' claims. They want a report on when a Leapfrog never event has occurred. They want to know what we're doing to incent the providers to not let these happen anymore ....

Most such policies closely mirrored CMS's list of HACs, the requirement for a POA indicator, and diagnosis-related group (DRG) grouper logic, though some include NQF "never events" or additional HACs, whereas others may include settings excluded from the CMS HAC-POA policy, including those with non-DRG payment systems. Most policies are reviewed and updated at least annually. A few insurers offer their own incentives for HAC prevention and reduction.

In the private sector, payment reductions (and in some cases the HACs) are negotiated as part of each hospital's contract. Payers can negotiate different contracts with various providers within a network. For example, five to six hospitals in a given market may have different reimbursement rates based on selected HACs. Some payers also differentiated payment reductions for HACs based on the degree of their preventability in recognition that some HACs can happen even with the best care. 
According to hospital staff, adoption of similar yet different policies by other payers created some negative, unintended consequences for some hospitals by causing confusion and difficulties in meeting inconsistent definitions, lists of HACs, documentation requirements, and payment systems.

Interviewees representing other payer organizations shared that the policy had minimal financial impact on their organizations, mostly attributed to the rarity of never events and because they serve younger and healthier populations compared with CMS beneficiaries. Some questioned whether the hospitals were billing for no-pay events altogether, given that several other payer policies did not require billing for HACs that were not present on admission initially. All interviewees representing other payers planned to continue their policies and hoped to expand their scope in terms of the list of HACs and the care setting in which they are applied.

\section{Effects on Patients}

All representatives of patient advocacy organizations were in strong support of the HAC-POA policy, sharing a firm position that payment is the only real incentive to improve patient safety across the hospitals: "any time you talk about money, you get the attention of people from the top." Consistent with views shared by the national health care organizations and hospital staff, patient advocates indicated that HAC-POA had a positive effect in getting hospital leadership attention on patient safety, even though payment reductions themselves did not constitute a "significant amount of [the] hospital bill." Patient advocates viewed adoption of the policy by other payers as a positive spillover effect.

\section{Policy Visibility to Patients and Patient Reporting}

Patient advocates were not aware of or had not observed such HAC-POA policy effects as denied access to care, use of data for litigation purposes, patients being responsible for "the bill," or different treatment by health care providers. In order to raise patient awareness, one patient advocate suggested that CMS create a national educational campaign about HAC-POA and send letters to patients who have experienced a harmful event to inform them that the hospital was not fully reimbursed.

Patient advocates emphasized the need for public reporting of HACs, such as through Hospital Compare, alongside patient-reported results. Combining such feedback with administrative data could provide a more accurate, holistic view of HAC incidence and nature:

There should be a place where patients can report things to CMS. ... Patient reporting is a tremendous recourse that has not been utilized and has been discouraged. There is a sort of idea that patients don't know what happened to them and sometimes that's true- they may not know why it happened, but they certainly know what happened to them.

Patient advocates also emphasized the absence of accurate HAC-specific information in patient records and death certificates, which contributes to inconsistencies in data and knowledge of HAC incidence as it is perceived by patients and reflected in vital statistics.

Patient advocates observed that reported POA rates were quite high and had concerns about validity of POA codes, which, in their observation, have been applied "too liberally", and that little effort has been put in place to identify organizations that might be misusing the application of POA codes.

One patient advocate shared a concern that the patient medical record has been used as a gold standard and that sometimes such records might be 
incomplete or altered by hospital staff. One patient advocate who collected feedback systematically from patients shared that $20 \%$ of patients believed that their medical records had been altered: "the accuracy and veracity of coding is a huge issue in the medical record itself because people just leave things out."

Patient advocates strongly encouraged CMS to conduct random audits to improve the accuracy of HAC-POA coding that include interviews with the patients. They suggested that in addition to accuracy of coding, such audits would allow tracking the origins of HACs across different care settings.

\section{Downstream Effects on the Public}

Patient advocates noted that financial HAC-POA penalties to hospitals do not take into account the true costs of HACs to CMS and taxpayers, because they capture only a small portion of costs limited to an isolated admission, and do not take into account readmissions and transitions to and from other care settings. One participant labeled these as "invisible" costs, because they have not been discussed or addressed despite the common knowledge that medical errors can result in medical bankruptcies and medical debts:

[This policy] only captures that one moment in time [of] the one hospitalization [that] CMS is paying for. I think we definitely need a broader perspective for the HACs; it's terrific to connect them with payment policies, but it's artificial and arbitrary to connect it only with that visit to the hospital .... If there's a readmission within 30 days, it should be recognized that it's something that went wrong with the previous admission.

One patient advocate further suggested that HAC-related downstream costs were a huge burden to all taxpayers, because many patients use Medicare further or turn to other public assistance programs in the aftermath of HACrelated complications:

Burden of these costs have shifted back onto the taxpayer. It's not just that Medicare is paying the medical bills, now we have public assistance programs because some of these patients can't afford the medical expenses from these complications .... One little complication can result in astronomical costs ....

Patient advocates desired CMS to expand the current list of HACs, especially previously considered conditions such as MethicillinResistant Staphylococcus Aureus (MRSA) and Clostridium Difficile Infections. They emphasized that preventability is not static and changes over time, with new, emerging evidence that should be taken into account through an on-going refinement of the policy.

\section{Conclusions and Discussion}

Understanding of HAC-POA policy effects on hospitals, patients, and other payers is important for maximizing its successful implementation and identifying opportunities for refinements. Unlike other studies, these findings offer a unique opportunity to assess the effects of the HACPOA policy during the first three years of policy implementation and across a broad variety of stakeholders. Additionally, the qualitative nature of our approach was conducive to identification of unintended consequences and allowed the emergence of the new topics, such as the downstream effects of policy to the public that were beyond the original conceptual structure of the study.

Prior to implementation, health care professionals and stakeholders cautioned that 
the policy could cause a number of unintended consequences; however, many of the unintended consequences anticipated prior to HAC-POA policy implementation did not materialize within the first three years that the policy was in place. One of the key changes to hospitals that did occur included a cultural shift involving attention, commitment, and support from hospital leadership for patient safety, confirming reports in the literature (Hoff et al., 2011; Krein, Kowalski, Hofer, \& Saint, 2012; McHugh, Van Dyke, et al., 2011; Wald et al., 2012). Other findings included the practice of hiring new staff to assure the accuracy of clinical documentation and POA oversight structures; increased time burden for physicians, nurses and coders; the need to upgrade or purchase new software; and the need to collaborate with hospital departments or staff that did not interface directly in the past (Uchida et al., 2011). While time burden was generally mentioned in conjunction with increased documentation requirements and coding reconciliation, some interviewees were concerned that the increased burden may be associated with shifts in resources away from other important areas. These shifts in attention have been reported elsewhere, both in terms of specific infection types (Hartmann et al., 2012; Lee, Hartmann, et al., 2012) as well as in daily activities (Stone et al., 2011). The data also support findings from California and elsewhere that hospitals are increasingly moving to adopt and adhere to evidence-based guidelines (Stone et al., 2011).

Concerns continue to be raised over the inaccuracy of administrative and claims data for identification of healthcare associated infections (Meddings et al., 2012). Despite increased hiring and training of coding and documentation specialists, as well as collaboration among clinical and administrative staff, the general level of mistrust in HAC-POA coding reported by some healthcare personnel during our interviews may indicate that these coding problems extend to other HACs as well.
Several interviewees observed that the key effect attributable to HAC-POA policy is that patient safety became a concern for an entire organization-in contrast to existing isolated improvement efforts geared toward a specific single unit-and for each organization (hospital and/or health care system) in the country, rather than a subset of leading organizational performers.

The majority of other payers adopted similar policies. Variations in definitions, lists of HACs, and documentation requirements caused some confusion and difficulties in meeting requirements of different payers.

Most patients have no knowledge of the HACPOA policy and would commonly not be aware that they have experienced a HAC. Therefore, policy effects on patients cannot be fully assessed at this time. Future refinements of the policy should seek to establish further consistency of definitions used by CMS and CDC, establish a stronger evidence base for HAC prevention, and increase visibility of the policy to patients.

This study has several limitations. Ongoing federal, national, and local patient safety improvement initiatives make it difficult to attribute efforts to improve HAC incidence to this policy alone. In addition, most interviews were conducted prior to posting HAC-POA data on Hospital Compare. We anticipate that different unintended consequences might emerge once data become publicly available and as hospitals, patients, and other payers gain further experience with the policy.

This study provides a comprehensive view of HAC-POA policy effects based on qualitative interviews of key stakeholders. Our approach allowed the emergence of unanticipated, but important, themes-such as the relationship between the policy and the "true" or downstream costs of HACs, as raised by patient advocatescompared to previously published, more narrowly 
focused studies. Although not all identified effects may be directly attributable to the HAC-POA policy alone, the findings suggest several fruitful avenues for future policy refinement and efforts to prevent hospital acquired conditions.

\section{Disclaimer}

This project was funded by the Centers for Medicare \& Medicaid Services under contract no. HHSM-500-200500029I. The statements contained in this report are solely those of the authors and do not necessarily reflect the views or policies of the Centers for Medicare \& Medicaid Services. RTI assumes responsibility for the accuracy and completeness of the information contained in this report.

\section{Correspondence}

Asta Sorensen, Research Triangle Institute, 3040 Cornwallis Rd., Research Triangle Park, North Carolina 27709, asorensen@rti.org, Tel. 919-541-1238, Fax. 919-541-7384

\section{References}

Brown, J., Doloresco, F., III, \& Mylotte, J. M. (2009).

"Never Events": Not Every Hospital-Acquired Infection is Preventable. Clinical Infectious Diseases, 49(5), 743-746. PubMed http://dx.doi. org/10.1086/604719

Hartmann, C. W., Hoff, T., Palmer, J. A., Wroe, P., Dutta-Linn, M. M., \& Lee, G. (2012). The Medicare Policy of Payment Adjustment for Health Care-Associated Infections: Perspectives on Potential Unintended Consequences. Medical Care Research and Review, 69(1), 45-61. PubMed http://dx.doi.org/10.1177/1077558711413606

Hoff, T., Hartmann, C. W., Soerensen, C., Wroe, P.,

Dutta-Linn, M., \& Lee, G. (2011). Making the CMS Payment Policy for Healthcare-Associated Infections Work: Organizational Factors that Matter. Journal of Healthcare Management, 56(5), 319-335. PubMed
Krein, S. L., Kowalski, C. P., Hofer, T. P., \& Saint, S. (2012). Preventing Hospital-Acquired Infections: A National Survey of Practices Reported by U.S. Hospitals in 2005 and 2009. Journal of General Internal Medicine, 27(7), 773-779. PubMed http://dx.doi.org/10.1007/ s11606-011-1935-y

Lee, G. M., Hartmann, C. W., Graham, D., Kassler, W., Dutta Linn, M., Krein, S., ... Jha, A. (2012). Perceived impact of the Medicare policy to adjust payment for health care-associated infections. American Journal of Infection Control, 40(4), 314-319. PubMed http://dx.doi.org/10.1016/j. ajic.2011.11.003

Lee, G. M., Kleinman, K., Soumerei, S., Tse, A., Cole, D., Fridkin, S. K., . . Jha, A. K. (2012). Effect of Nonpayment for Preventable Infections in U.S. Hospitals. The New England Journal of Medicine, 367(15), 1428-1437. PubMed http://dx.doi.org/10.1056/NEJMsa1202419

McHugh, M., Martin, T. C., Orwat, J., \& Dyke, K. V. (2011). Medicare's Policy to Limit Payment for Hospital-Acquired Conditions: The Impact on Safety Net Providers. Journal of Health Care for the Poor and Underserved, 22(2), 638-647. PubMed http://dx.doi.org/10.1353/ hpu.2011.0058

McHugh, M., Van Dyke, K., Osei-Anto, A., \& Haque, A. (2011). Medicare's Payment Policy for Hospital-Acquired Conditions: Perspectives of Administrators from Safety Net Hospitals. Medical Care Research and Review, 68(6), 667-682. PubMed http://dx.doi. org/10.1177/1077558711408326

McKibben, L., Horan, T., Tokars, J. I., Fowler, G., Cardo,D.M.,Pearson,M.L....HealthcareInfection Control Practices Advisory Committee, (2005). 
Guidance on Public Reporting of HealthcareAssociated Infections: Recommendations of the Healthcare Infection Control Practices Advisory Committee. American Journal of Infection Control, 33(4), 217-226. PubMed http://dx.doi. org/10.1016/j.ajic.2005.04.001

Meddings, J. A., Reichert, H., Rogers, M. A., Saint, S., Stephansky, J., \& McMahon, L. F. (2012). Effect of Nonpayment for Hospital-Acquired, CatheterAssociated Urinary Tract Infection: A Statewide Analysis. Annals of Internal Medicine, 157(5),305312. PubMed http://dx.doi.org/10.7326/00034819-157-5-201209040-00003

Miles, M. B., \& Huberman, M. A. (1994). Qualitative Data Analysis: an Expanded Sourcebook, 2nd edition., SAGE Publications.

Parish, J. (2008). Advice on the CMS Mandate: Its Impact and Reducing 'Preventable Errors.' Bureau of National Affairs' Medicare Report, 19(43).

Patton, M. Q. (1990). Qualitative Evaluation and Research Methods, 2nd edition., SAGE Publications.

Peasah, S. K., McKay, N. L., Harman, J. S., Al-Amin, M., \& Cook, R. L. (2013). Medicare Non-Payment of Hospital-Acquired Infections: Infection Rates Three Years Post Implementation. Medicare \& Medicaid Research Review, 3(3), E1-E16. PubMed http://dx.doi.org/10.5600/mmrr.003.03.a08

Rosenstein, A. H., O'Daniel, M., White, S., \& Taylor, K. (2009). Medicare's Value-Based Payment Initiatives: Impact on and Implications for Improving Physician Documentation and Coding. American Journal of Medical Quality, 24(3), 250-258. PubMed http://dx.doi. org/10.1177/1062860609332511
Saint, S., Meddings, J. A., Calfee, D., Kowalski, C. P., \& Krein, S. L. (2009). Catheter-Associated Urinary Tract Infection and the Medicare Rule Changes. Annals of Internal Medicine, 150(12), 877-884. PubMed http://dx.doi.org/10.7326/0003-4819150-12-200906160-00013

Stone, P. W., Glied, S. A., McNair, P. D., Matthes, N., Cohen, B., Landers, T. F., \& Larson, E. L. (2010). CMS Changes in Reimbursement for HAIs: Setting a Research Agenda. Medical Care, 48(5), 433-439. PubMed http://dx.doi.org/10.1097/ MLR.0b013e3181d5fb3f

Stone, P. W., Pogorzelska, M., Graham, D., Jia, H., Uchida, M., \& Larson, E. L. (2011). California Hospitals Response to State and Federal Policies Related to Health Care-Associated Infections. Policy, Politics \& Nursing Practice, 12(2), 73-81. doi:

Uchida, M., Stone, P. W., Conway, L. J., Pogorzelska, M., Larson, E. L., \& Raveis, V. H. (2011). Exploring Infection Prevention: Policy Implications from A Qualitative Study. Policy Politics in Nursing Practice 12(2), 82-89. doi:

Wald, H., Richard, A., Dickson, V. V., \& Capezuti, E. (2012). Chief Nursing Officers' Perspectives on Medicare's Hospital-Acquired Conditions Non-payment Policy: Implications for Policy Design and Implementation. Implement Science, 7(78). doi:

Wald, H. L., \& Kramer, A. M. (2007). Nonpayment for Harms Resulting from Medical Care: CatheterAssociated Urinary Tract Infections. Journal of the American Medical Association, 298(23), 2782-2784. PubMed http://dx.doi.org/10.1001/ jama.298.23.2782 